\title{
Optical Properties of Organic Materials for Optical Communication Devices
}

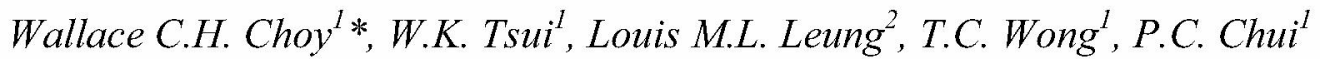 \\ ${ }^{1}$ Department of Electrical and Electronic Engineering, the University of Hong Kong, Pokfulam Road, \\ Hong Kong SAR, PRC. \\ ${ }^{2}$ Centre for Advanced Luminescence Materials, Hong Kong Baptist University, \\ Kowloon Tong, Kowloon, Hong Kong SAR, PRC. \\ *chchoy@eee.hku.hk
}

\begin{abstract}
Recently, organic materials have been considered as a potential candidate for making optoelectronic devices. In order to optimize the performance of the optoelectronic devices, the absolute refractive index and absorption coefficient of the organic materials have to be determined. In this article, the absolute optical properties of two novel bipolar organic materials will be investigated through ellipsometry and modeling using Lorentz Oscillator model and stimulated annealing algorithm.
\end{abstract}

\section{INTRODUCTION}

Since the first demonstration of low-voltage driven organic light emitting diodes (OLEDs) in 1987 [1], OLED has been widely investigated for use in colour display, signaling and light illustration. It has also been proposed to use OLEDs for local optical communications. It has been a well-known fact that the performance of optical devices is very sensitive to the optical properties of the materials including refractive index and absorption coefficient [2].

In this article, the absolute optical properties of organic materials will be studied through ellipsometry and modeling. In the model, simulated annealing (SA) algorithm will be utilized to determine the absolute optical properties through Lorentz Oscillators model.

\section{MODELING AND EXPERIMENT}

In order to optimize the optical devices for optical communication, the absolute optical properties of the materials were determined by ellipsometry and modeling through SA algorithm using Lorentz Oscillator model. The complex dielectric constant of the materials is written as:

$$
\varepsilon=\varepsilon_{1}+i \varepsilon_{2}
$$

The real and imaginary parts of complex refractive index are $\mathbf{n}$ and $\mathrm{k}$ respectively and can be described as

$$
\begin{aligned}
& n=\left[0.5\left(\varepsilon_{1}+\left(\varepsilon_{1}^{2}+\varepsilon_{2}^{2}\right)\right)^{1 / 2}\right]^{1 / 2} \text { and } \\
& k=\left[0.5\left(-\varepsilon_{1}+\left(\varepsilon_{1}^{2}+\varepsilon_{2}^{2}\right)\right)^{1 / 2}\right]^{1 / 2}
\end{aligned}
$$

It should be noted that the imaginary part of refractive index is the so-called extinction coefficient. The absorption coefficient is proportional to the extinction coefficient:

$$
\alpha=\frac{4 \pi}{\lambda} k
$$

The absolute refractive index and absorption coefficient can be determined from the experimental results obtained from ellipsometry. The measured ellipsometry parameters are $\tan _{\text {expt }}(\Psi)$ and $\cos _{\text {expt }}(\Delta)$. The theoretical tan $\operatorname{theo}_{\text {the }}(\Psi)$ and $\cos _{\text {theo }}(\Delta)$ can be determined from Fresnel Reflection coefficient $R_{p}$ and $R_{s}$ for $p$ - and s- polarized light [3].

$\tan _{\text {theo }}(\Psi) \mathrm{e}^{\mathrm{i} \Delta}=\mathrm{R}_{\mathrm{p}} / \mathrm{R}_{\mathrm{s}}$

Fresnel Reflection coefficient and thus $\tan _{\text {theo }}(\Psi)$ and $\cos _{\text {theo }}(\Delta)$ can be determined from the refractive index and absorption coefficient of the layer structures through transfer matrix method [4]. By utilizing a simulated annealing algorithm [5], the absolute refractive index and absorption coefficient can be obtained when the objective function below is within the tolerance.

$$
E=\sum\left[\left(\tan _{\text {expt }}(\Psi)-\tan _{\text {theo }}(\Psi)\right)^{2}+\left(\cos _{\text {expt }}(\Delta)-\cos _{\text {thoe }}(\Delta)\right)^{2}\right]
$$

A flowchart of the modeling is shown in Fig. 1. 
The investigated organic materials are phenyl-N-(4-(5phenyl-1,3,4-oxadiazol-2-yl)phenyl)phenylamine (POT) and tris(4-(5-phenyl-1,3,4-oxadiazol-2-yl)phenyl)amine $\left(\mathrm{N}(\mathrm{OXD})_{3}\right)$. They were potential bipolar organic materials which can simultaneously function as electron and hole transport materials. The novel materials were synthesized by Leung's group in Hong Kong Baptist University. The measurement and theoretical calculation were conducted in the University of Hong Kong. The materials were purified by gradient sublimation prior to thin-film coating. The deposition rate was typically $1.0-$ $2.0 \AA / \mathrm{s}$ and the substrate was $\mathrm{Si}$. The evaporation chamber was operated at $\sim 10^{-6}$ Torr. Film thickness was monitored in situ using the quartz crystal monitor and ex situ by a stylus profilometer (Tencor $\alpha$-step 500). The thickness of POT and $\mathrm{N}(\mathrm{OXD})_{3}$ are $40.6 \mathrm{~nm}$ and $68.6 \mathrm{~nm}$ respectively.

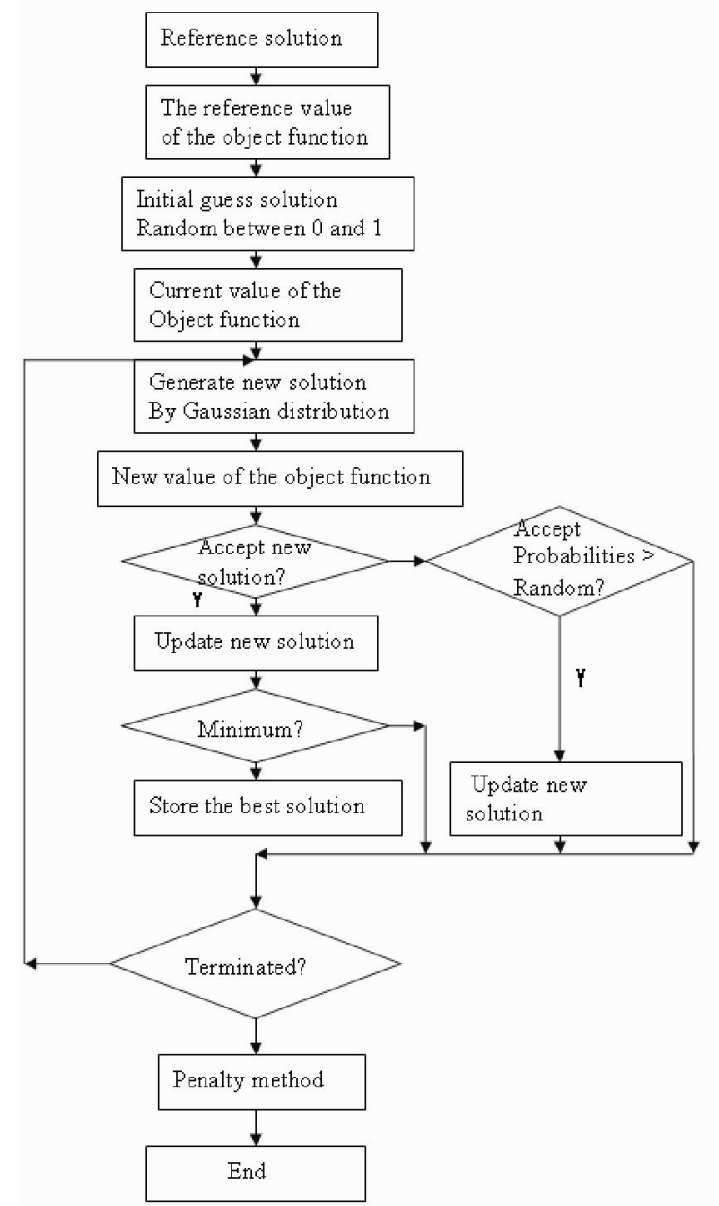

Fig. 1. Flowchart of the model of absolute optical properties.

\section{RESULTS}

By using the Lorentz Oscillator model, the complex dielectric constant can be described as

$$
\begin{aligned}
& \varepsilon_{1}(\omega)=\varepsilon_{\infty}+\sum_{j=1}^{k} \frac{F_{j}\left(\omega_{j}^{2}-\omega^{2}\right)}{\left(\omega^{2}-\omega_{j}^{2}\right)^{2}+\left(\omega \Gamma_{j}\right)^{2}}, \text { and } \\
& \varepsilon_{2}(\omega)=\sum_{j=1}^{k} \frac{F_{j} \omega \Gamma_{j}}{\left(\omega^{2}-\omega_{j}^{2}\right)^{2}+\left(\omega \Gamma_{j}\right)^{2}}
\end{aligned}
$$

where $\mathrm{k}$ is the total number of interband transitions at frequency $\omega_{j}, 1 / \Gamma_{j}$ is the lifetime and $F_{j}=\omega_{j}{ }^{2}$ is the parameter associated with oscillator strength $f_{j}$. The parameters and thus the complex dielectric constant will be determined from the model as shown in Fig. 1.

The experimental and theoretical $\tan (\Psi)$ and $\cos (\Delta)$ of POT and the absolute refractive index and absorption coefficient are shown in Fig. 2. The $\tan _{\text {expt }}(\Psi)$ and $\cos _{\text {expt }}(\Delta)$ can be fitted using the parameters of the complex dielectric constant. The fitted parameters are shown in Table 1 when $\omega_{1 \infty}=2.1486$.

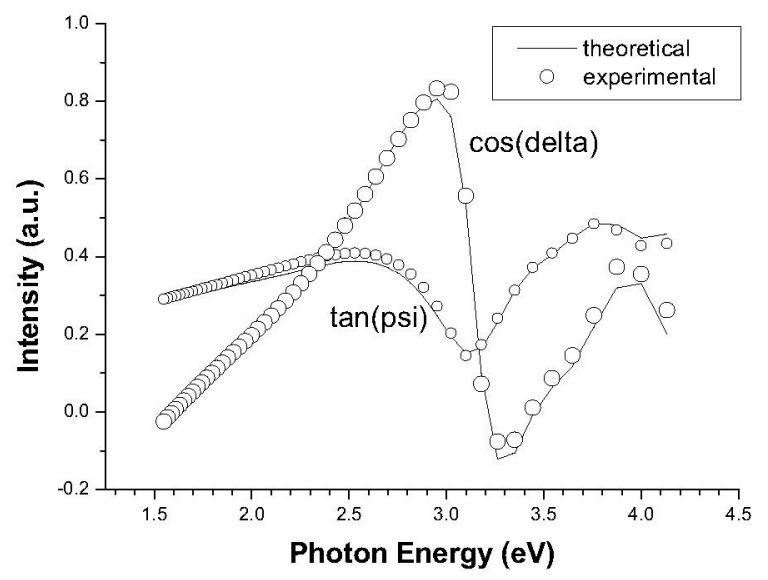

Fig. 2(a) The experimental and theoretical results of $\tan (\Psi)$ and $\cos (\Delta)$ of POT.

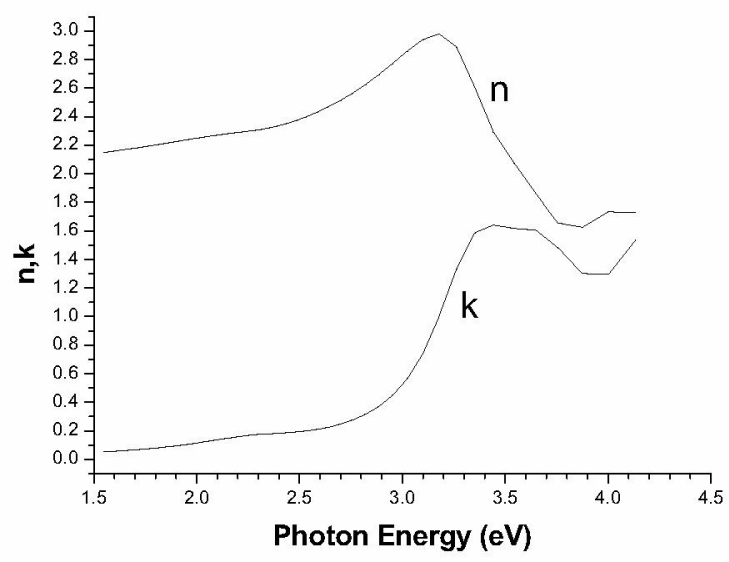


Fig 2(b). The absolute refractive index and absorption coefficient of POT

\begin{tabular}{|l|l|l|l|}
\hline $\mathbf{j}$ & $\mathrm{F}_{\mathrm{j}}(\mathrm{eV})^{2}$ & $\Gamma_{\mathrm{j}}(\mathrm{eV})$ & $\omega_{\mathrm{j}}(\mathrm{eV})$ \\
\hline 1 & 0.5279 & 0.7102 & 2.2814 \\
\hline 2 & 10.0917 & 0.455185 & 3.32122 \\
\hline 3 & 5.1889 & 0.47624 & 3.6274 \\
\hline 4 & 10.2043 & 0.540227 & 4.201 \\
\hline
\end{tabular}

Table 1. Fitted parameters of POT

Similarly, the experimental $\tan (\Psi)$ and $\cos (\Delta)$ of $n(O X D)_{3}$ are also fitted as shown in Fig. 3(a). The absolute refractive index and absorption coefficient and the fitted parameters are shown in Fig. 3(b) and Table 2 respectively when $\omega_{1 \infty}=2.4411$.

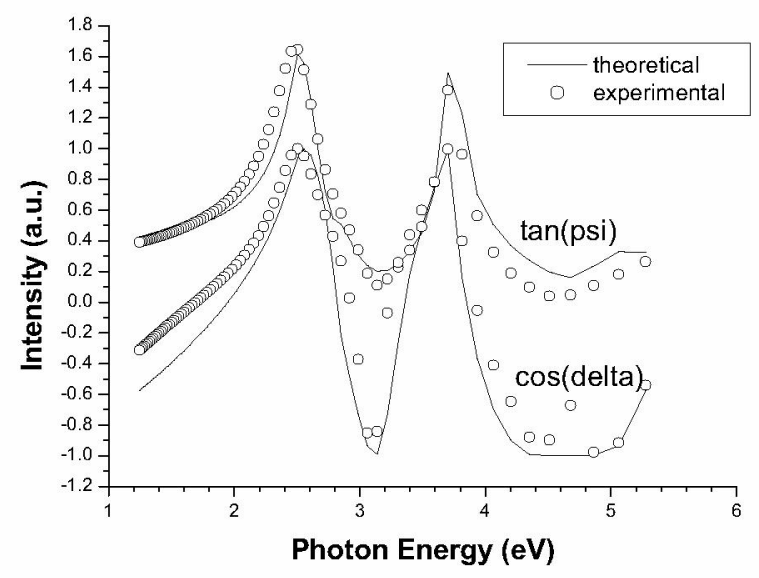

Fig. 3(a) The experimental and theoretical results of $\tan (\Psi)$ and $\cos (\Delta)$ of $N(O X D)_{3}$.

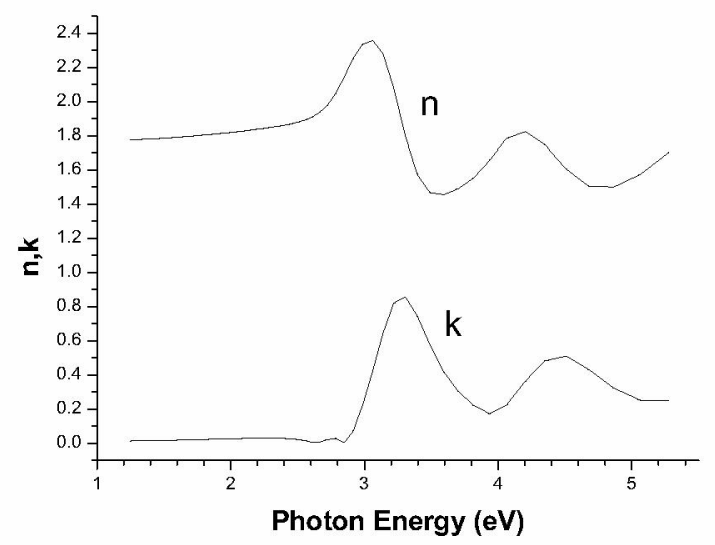

Fig. 3(b) The absolute refractive index and absorption coefficient of $\mathrm{N}(\mathrm{OXD})_{3}$.

\begin{tabular}{|l|l|l|l|}
\hline $\mathbf{j}$ & $\mathrm{F}_{\mathrm{j}}(\mathrm{eV})^{2}$ & $\Gamma_{\mathrm{j}}(\mathrm{eV})$ & $\omega_{\mathrm{j}}(\mathrm{eV})$ \\
\hline 1 & -1.7766 & 0.5041 & 2.8536 \\
\hline 2 & 5.9138 & 0.4694 & 3.2336 \\
\hline 3 & -1.4108 & 0.5259 & 3.9839 \\
\hline 4 & 6.4286 & 0.9068 & 4.4943 \\
\hline
\end{tabular}

Table 1. Fitted parameters of N(OXD) 3

The results show that four oscillators are enough for fitting the optical properties of both POT and N(OXD) ${ }_{3}$. The photon energy of the extinction coefficient edge and thus the absorption edge of $\mathrm{N}(\mathrm{OXD})_{3}$ is smaller than that of POT. One of the reasons is that molecule weight of $\mathrm{N}(\mathrm{OXD})_{3}$ is larger than that of POT. There are two clear absorption peaks for $\mathrm{N}(\mathrm{OXD})_{3}$ when there is only one peak for POT. It should be noted that the peaks of extinction coefficient are the tuning point of refractive index because extinction coefficient and refractive index can be related by Kramer Krönig relationship.

\section{CONCLUSION}

The absolute refractive index and absorption coefficient of two novel dipolar organic materials have been determined using ellipsometry and modeling using Lorentz Oscillator model using stimulated annealing algorithm. The optical properties are important for use of the organic materials in optoelectronic devices. The method can also be used to determine the absolute optical properties of other inorganic materials such as semiconductors.

Acknowledgement

The authors would like to acknowledge the support of UDF grant and seed funding of The University of Hong Kong. P.C Chui would like to acknowledge the support of the small project funding.

\section{REFERENCES}

[1] C.W. Tang, S.A. Vanslyke, "Organic electroluminescent diodes", Appl. Phys. Let., vol. 51, pp.913-915, 1987.

[2] W.C.H. Choy, "Optical Properties of InGaAs/InAlAs Diffused Double Quantum Wells", J. Appl. Phys., vol. 87, pp. 2956-2966, 2000.

[3] John A. Woollam, Blaine Johs, Craig M. Herzinger, James Hilfiker, Ron Synowicki, and Corey L. 
Bungay, "Overview of Variable Angle Spectroscopic Ellipsometry (VASE), Part I: Basic Theory and Typical Applications", Critical Rev. of Optical Sci. and Technol., vol. CR72, pp. 3-28, 2002.

[4] W.C.H. Choy and E.H. Li, "The applications of interdiffused quantum well in normally-on electroabsorptive Fabry-Perot reflection modulator", IEEE J. Quantum Electron., vol. 33, pp. 382-393, 1997.

[5] N. Metropolis, A.W. Rosenbluth, M. N. Rosenbluth, A.H. Teller, and E. Teller, "Equations of State Calculations by Fast Computing Machines", J. Chem. Phys. vol. 21, pp. 1087-1092, 1958. 